\title{
Answer to the Letter to the Editor of Marco A. Marino et al. concerning "Circumferential fusion using a custom-made screw in the management of high-grade spondylolisthesis" by Jouve JL, et al. (2014) Eur Spine J; 23(Suppl. 4):S457-S462
}

\author{
J. L. Jouve ${ }^{1}$ G. Bollini ${ }^{1}$
}

Received: 7 May 2015/Revised: 7 May 2015/Accepted: 7 May 2015/Published online: 19 May 2015

(c) Springer-Verlag Berlin Heidelberg 2015

We have read with great interest your letter concerning our article entitled "an original" technique using a posterioronly approach to achieve a circumferential fusion with a custom-made screw.

You stated that our technique is not "original" having being published by you in 2003 .

In your article, you mentioned "Posterior decompression, partial reduction, pedicular fixation and posterior lumbar interbody fusion using a trans-sacral titanium cage".

You used an approach from L2 to the sacral ala and you used two Harrington rods between L2 and the sacral alae to partially reduce the slip. You then performed a L4/L5 PLIF and you mentioned that "none of your patients had undergone sacral dome resection or L5/S1 discectomy."

We do not address the L4/L5 level in most of our cases.

We do not believe that it is necessary to add any additional "Rod and pedicle screws constructs" to our screw for two reasons:

J. L. Jouve

jean-luc.jouve@ap-hm.fr

1 Service de Chirurgie Orthopédique, Hôpital Timone Enfants, 264 Rue St-Pierre, 13385 Marseille Cedex 5, France
The first one is that we do not try to obtain any additional slip reduction except the one obtained in a prone position with flexed hips and knees and under muscle's drugs release.

The second one is that our screw is strong enough to fight against the shear forces at L5/S1 level.

At the beginning of our experience, we used fibular graft, then hollow screws and both of them bended and broke.

To summarize, our screw is the key point of our technique, strong enough to neutralize, after partial spontaneous slip reduction, all the shear forces at L5/S1 level allowing a limited L5/S1 circumferential arthrodesis with a PLIF done after L5/S1 disc excision and bone grafting (using bone graft harvested from the iliac crest).

We apologize having not quoted your article but we think that the two techniques are quite different and that the term "original" has to be kept.

Conflict of interest None. 